\title{
Statine wirksam in der Primärprävention
}

\author{
Eine große Metaanalyse zeigt, dass zur Primärprävention verschriebene Statine die Raten der gefähr- \\ lichsten vaskulären Ereignisse signifikant reduzieren - auch bei Cholesterinspiegeln $<200 \mathrm{mg} / \mathrm{dl}$.
}

\begin{abstract}
Erhöhte Cholesterinwerte sind ein wichtiger Risikofaktor für die koronare Herzerkrankung (KHK) und den Herzinfarkt. Hinsichtlich des Schlaganfalls gelten sie als weniger relevant. Umstritten ist die Frage, ob Statine auch als Primärprävention wirksam sind. Eine Gruppe von US-Forschern analysierte daher 19 randomisierte Studien mit insgesamt 71.344 Teilnehmern. Verglichen wurden jeweils Statine mit Placebo. Endpunkte waren die Gesamtsterblichkeit, die kardiovaskuläre Mortalität sowie Herzinfarkt und Schlaganfall.

Patienten mit Hyperlipidämie waren in sechs der Studien eingeschlossen, solche mit Schlaganfall in dreien, Diabetiker in vieren und Hypertoniker in zweien. Am häufigsten wurden Simvastatin und Atorvastatin untersucht.
\end{abstract}

Für die Gesamtsterblichkeit zeigte sich unter Statinen eine signifikante Risikoreduktion um 14\%. Die kardiovaskuläre Sterblichkeit war um 31\% reduziert, die Schlaganfallrate um 29\% und die Herzinfarktrate um 35\%. Für den kombinierten Endpunkt Herzinfarkt, Schlaganfall und kardiovaskulärer Tod betrug die Risikoreduktion 30\%.

Die Therapieeffekte waren über demografische und klinische Subgruppen hinweg identisch. Der Nutzen war umso höher, je höher der Cholesterinspiegel war. Trotzdem war er auch bei Werten $<200 \mathrm{mg} / \mathrm{dl}$ nachweisbar. Leberschäden wurden unter der Statintherapie nicht beobachtet. Es ergab sich auch kein erhöhtes Diabetesrisiko. Keine der Studien untersuchte eine am Cholesterinspiegel orientierte, titrierte Therapie.
- Chou R, Dana T, Blazina l et al. Statin use for the prevention of cardiovascular disease in adults: a systematic review for the U.S. Preventive Services Task Force. JAMA. 2016;316:2008-24

\section{KOMMENTAR}

Diese große Metaanalyse zeigt, dass die Patienten über einen weiten Bereich von Cholesterinspiegeln von einer Behandlung mit Statinen profitieren. Dies gilt sowohl für die Primär-als auch für die Sekundärprävention und für alle vaskulären Endpunkte. Herzinfarkte werden etwas besser verhindert als Schlaganfälle. Weiterhin ist leider nicht geklärt, ob alle Patienten dieselbe Dosis eines Statins erhalten sollen oder ob ein bestimmter LDL-Cholesterin-Wert angestrebt werden sollte. Daher werden in den neuen Leitlinien zur Hypercholesterinämie auch keine Zielwerte angegeben.

Prof. Dr. med. H.-C. Diener

\section{Mandelentzündung und Ausschlag am ganzen Körper}

Ein 20-jähriger Mann suchte wegen geschwollener Mandeln, Rachenschmerzen, Fieber, Schüttelfrost und eines Exanthems den Hausarzt auf. Der Ausschlag hatte sich vom Bauch auf Brust und Rücken, später auch auf Arme, Beine und Gesicht ausgebreitet. Die Frage nach Allergien und der Einnahme neuer Medikamente verneinte er. Bei der Untersuchung zeigte sich eine Tonsillitis mit ausgedehnten grauweißen Belägen (Abb. A), eine Erdbeerzunge und schmerzhaft vergrößerte Lymphknoten im Zervikalbereich. Das ausgedehnte Exanthem war feinfleckig und makulopapulös, die Haut fühlte sich samten an (Abb. B, linke Flanke). Im Nacken und im Bereich der rechten Flanke fanden sich linear angeordnete Petechien. Ein Schnelltest auf $\beta$-hämolysierende Streptokokken war positiv. Die Befunde führten zur Diagnose eines Scharlachs.

Das Exanthem beim Scharlach ist eine Hypersensitivität vom verzögerten Typ gegen ein Exotoxin und tritt daher bei Personen auf, die früher bereits einmal gegenüber Streptococcus pyogenes exponiert waren. Der junge Mann wurde mit Penicillin V behandelt, worauf sich die Symptomatik innerhalb von drei Tagen zurückbildete.

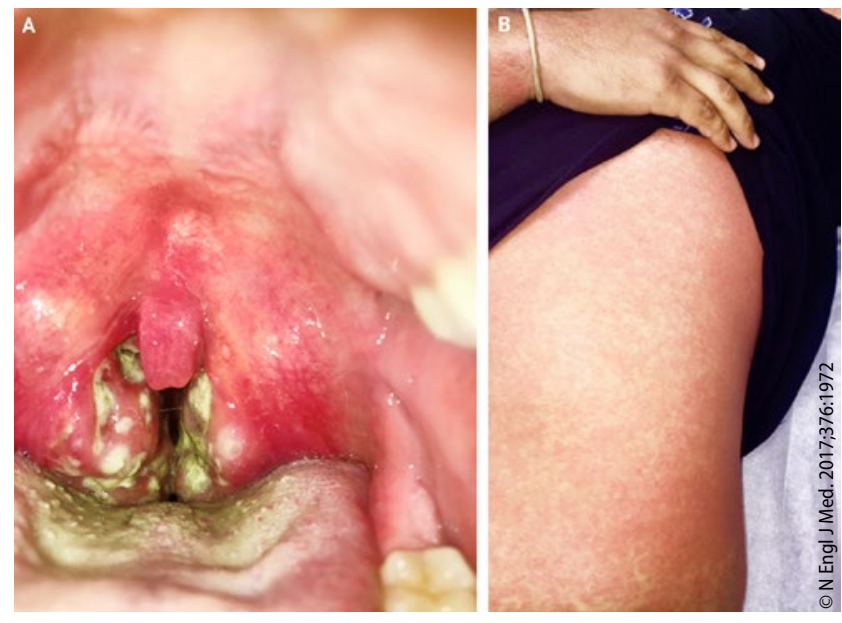

A: Ausgeprägte Tonsillitis. B: Feinfleckiges, makulopapulöses Exanthem (hier: linke Flanke).

- Brinker A. Scarlet fever. N Engl J Med. 2017;376:1972 